\title{
Anestesia peridural con paciente despierto para cirugía de tórax
}

\section{Epidural anesthesia in awake thoracic surgery}

Indira Fabiana Cujiño, MD. ${ }^{(1)}$; Mauricio Velásquez, MD. ${ }^{(2)}$

\section{RESUMEN}

La anestesia general complementada con la peridural, es en la actualidad la técnica de elección en los pacientes de cirugia de tórax; sin embargo, nuevos estudios sugieren que la anestesia peridural sola puede tener algunos beneficios. Se realizó un estudio prospectivo que incluyó todos los pacientes sometidos a toracoscopia para cualquier procedimiento torácico y que se hicieran con una técnica de anestesia peridural con el paciente despierto. Se incluyeron veinte pacientes que fueron seguidos durante toda su hospitalización en búsqueda de eventos adversos cardiacos, pulmonares, renales o hidroelectroliticos, muerte y los relacionados con el manejo del dolor postoperatorio. Se hicieron pleurodesis con talco, decorticaciones y cuñas pulmonares. Cuatro pacientes presentaron alguna complicación y tres fallecieron debido a su enfermedad de base. Este estudio sugiere que la anestesia peridural con el paciente despierto es una técnica segura para los procedimientos por toracoscopia y que se asocia con una incidencia baja de complicaciones respiratorias y dolor postquirúrgico. Se requieren más estudios clínicos que corroboren esta observación.

Palabras clave: anestesia peridural, cirugia torácica, toracoscopia.

\section{ABSTRACT}

General anesthesia combined with epidural anesthesia is currently the technique of choice in patients undergoing thoracic surgery. However, new studies suggest that epidural anesthesia alone could have some benefits. A prospective study was done which included all patients who underwent thoracoscopy for any thoracic procedure performed with epidural anesthesia with the patient awake. Twenty (20) patients were included and followed up during their whole hospital stay in search for adverse cardiac, pulmonary, renal, or hydroelectrolytic adverse effects, death, or events related with postoperative pain management. Talc pleurodesis, decortications, and wedge resections were performed. Four patients had any complication and 3 patients died due to their basal illness. This study suggests that awake epidural anesthesia is a safe technique for thoracoscopic procedures. It is associated with low rates of respiratory complications and postoperative pain. Further clinical studies are needed in order to confirm this observation.

Keywords: epidural anesthesia, thoracic surgery, thoracoscopy.

Rev Colomb Neumol 2013; 24 (2):76-79

\section{INTRODUCCIÓN}

Los progresos en ventilación pulmonar y el desarrollo de las técnicas anestésicas, han permitido el desarrollo concomitante de la cirugía de tórax (1-3).
La necesidad de colapso pulmonar en cirugía, estimuló el desarrollo de dispositivos que permitieran aislar un pulmón de forma ventilatoria. Estudios posteriores permitieron conocer la fisiología de la ventilación unipulmonar, las indicaciones de la separación pulmonar

(1) Médica Anestesióloga, Fundación Valle del Lili, Cali, Colombia.
(2) Médico Cirujano de Tórax, Fundación Valle del Lili, Cali, Colombia.

Correspondencia: Doctora Indira Fabiana Cujiño. Correo electrónico: indirafcuji@yahoo.com

Recibido: septiembre 8 de 2012. Aceptado: noviembre 5 de 2012. 
y las técnicas para lograr este propósito (2). Hasta el momento, la anestesia general sola o en combinación con anestesia peridural se considera la mejor opción en el manejo anestésico de los pacientes de cirugia torácica $(2,3)$.

Las ventajas de la anestesia peridural torácica son ampliamente conocidas en cirugia de tórax. Existe evidencia a favor de la técnica neuroaxial peridural y su relación con menor sangrado intraoperatorio, mejor manejo del dolor postoperatorio, inicio temprano de la deambulación y de la vía oral, atenuación de la respuesta al estrés quirúrgico, disminución de la frecuencia cardiaca y las arritmias relacionadas con la manipulación intraoperatoria, mejoría en los determinantes del flujo sanguíneo miocárdico y la función ventricular izquierda, extubación temprana, disminución de las complicaciones trombóticas postoperatorias, disminución del riesgo de infecciones postoperatorias y mejoría en la función pulmonar postoperatoria (4-11).

En la búsqueda por evitar malos desenlaces relacionados con la anestesia general, como las complicaciones de la vía aérea por manipulación de la misma, mayor riesgo de neumonia, posibilidad de injuria pulmonar por la ventilación mecánica y aparición de atelectasias en el pulmón dependiente, se han publicado estudios en los que se ha utilizado la anestesia peridural para procedimientos como cirugía de bypass coronario, timectomía transesternal y cirugia de mama, con resultados benéficos a favor de esta técnica anestésica. $(5,9,12-14)$. En cirugia de tórax, especificamente, sólo dos grupos en el mundo han mostrado la utilización de anestesia peridural con el paciente despierto para procedimientos quirúrgicos en tórax $(5,15,16)$.

Dado lo anterior, se realizó un estudio que analizará diferentes desenlaces postoperatorios en pacientes con distintas patologías y procedimientos hechos con técnica peridural con el paciente despierto (APPD).

\section{MATERIALES Y MÉTODOS}

Se llevó a cabo un estudio descriptivo prospectivo, desde el $1^{\circ}$. de enero de 2010 al 31 de diciembre de 2010 , en el que se incluyeron todos los pacientes mayores de 18 años, programados para cirugia de tórax, que fuera realizada con APPD durante el período de estudio.

Para el registro prospectivo de los datos se diseñó un instrumento para tal fin, en el que se registraban los datos del perioperatorio temprano así como aquellos relacionados con los desenlaces de interés hasta los primeros treinta dias del postoperatorio.

Todos los procedimientos estuvieron a cargo de un grupo de anestesiólogos entrenados en anestesia para cirugia de tórax, quienes estandarizaron previamente el manejo intraoperatorio de la técnica anestésica utilizada. Ninguno de los anestesiólogos participó en el manejo postoperatorio o en la toma de decisiones durante la estancia en la unidad de cuidado intensivo o pisos, asi como en la valoración de los desenlaces postoperatorios tempranos, los cuales fueron registrados por una persona previamente entrenada para tal fin.

Para la técnica APPD, se administró $1 \mathrm{mg}$ de midazolam por via intravenosa y luego se insertó un catéter peridural \#18 en el espacio de T3-T4 por técnica de pérdida de resistencia. Una vez pasado el catéter y previa verificación de su adecuada posición, se pasaron $8 \mathrm{~mL}$ de bupivacaina al $0,5 \%$, con epinefrina. Se ubicó al paciente en decúbito lateral, se utilizó un sistema de Ventury al $50 \%$ para la administración de oxígeno y se vigiló el procedimiento con monitoría básica (incluida capnografía a través de una de las fosas nasales del paciente). El nivel de bloqueo se evaluó de manera constante para mantener niveles de bloqueo sensitivo entre T1 y T7 aplicando bolos adicionales de 2-4 mL más de bupivacaina al $0,5 \%$ si se consideraba necesario. Luego de cirugia todos los pacientes permanecieron con el catéter peridural por 24 horas más en espera de la decisión del uso o no de analgesia peridural continua por el grupo de clínica del dolor.

Todos los procedimientos fueron realizados por el mismo cirujano, quien utilizó un solo puerto de toracoscopia en el quinto espacio intercostal con línea axilar anterior, de 20 a 30 milímetros de longitud, por el que se introdujo simultáneamente la cámara y los instrumentos de trabajo de acuerdo con cada caso. Al final de la cirugia se dejó un tubo de toracostomia $28 \mathrm{Fr}$. Al terminar el procedimiento todos los pacientes se trasladaron a la unidad de cuidados postanestésicos, en donde eran evaluados por el grupo de cuidado intensivo quienes decidian la mejor ubicación de éstos para su cuidado postoperatorio.

El análisis estadístico se realizó con el software estadistico STATA 10 (StataCorp 4905 Lakeway Dr. USA). Las variables categóricas se muestran como frecuencias absolutas y frecuencias relativas y las variables cuantitativas se presentan como medidas de tendencia central y dispersión (promedio, DS, si se cumplian los criterios de normalidad) y como medianas y rangos intercuartílicos para los datos dispersos.

Dado que este estudio era de tipo observacional, se decidió, desde el inicio del diseño, no realizar un 
consentimiento informado específico para la investigación adicional a los consentimientos de anestesiologia y cirugia que por ley se exigen.

\section{RESULTADOS}

Se incluyeron 20 pacientes, 14 mujeres y 6 hombres, con una edad promedio de 72 (41-95) años.

En la tabla 1 se muestran los datos descriptivos de las cirugias y los diagnósticos de los pacientes intervenidos. Los procedimientos realizados fueron pleurodesis con talco, resecciones pulmonares en cuña y decorticaciones.

Los datos de los resultados y el manejo postoperatorio (tabla 2) muestran los dias de necesidad de cuidado intensivo, de ventilación mecánica, los pacientes que necesitaron el catéter peridural en el postoperatorio inmediato, los días de su uso, y finalmente los dias de hospitalización.

Tres pacientes fallecieron, los tres tenian diagnóstico de cáncer y el procedimiento que se les realizó fue una pleurodesis con talco; sin embargo la muerte no se relacionó con el procedimiento y, en dos casos se debió a enfermedad avanzada y en el otro a tromboembolismo pulmonar. Las demás complicaciones se registran en la tabla 3.

\section{DISCUSIÓN}

La anestesia general sola o combinada con anestesia peridural es hoy la técnica de elección en la mayoria de pacientes que requieren algún tipo de cirugia torácica. En este estudio se muestra una serie de pacientes con diferentes diagnósticos y procedimientos realizados bajo APPD.

Este estudio muestra que la APPD es una técnica segura incluso en pacientes ASA 3-4, con tiempos quirúrgicos y anestésicos aceptables si se compara con la duración de una cirugia bajo anestesia general. Los tiempos de hospitalización pudieran considerarse un poco prolongados para el tipo de procedimientos realizados; sin embargo están justificados por la clase funcional de estos pacientes. En cuanto a los tiempos totales de hospitalización prolongados, tienen soporte en el hecho de que en nuestra institución es frecuente que los pacientes con cáncer de cualquier tipo queden a cargo del Servicio de Oncologia, donde inician estudios y tratamientos oncológicos mientras permanecen hospitalizados. También se demostró que el EVA (escala visual análoga de dolor) inmediato y a las 24 horas postoperatorias, fue menor de 5 y que no hubo necesidad de utilizar analgésicos opioides, lo cual permite, como lo menciona Waurick, (17) que el paciente pueda deambular y realizar actividades como comer y levantarse al baño más pronto, y disminuir asi los riesgos de trombosis venosa profunda, tromboembolismo pulmonar, atelectasias pulmonares, neumonía, estreñimiento e infección urinaria.

La mitad de los pacientes del estudio fueron a la unidad de cuidado intensivo y sólo cinco requirieron algún tipo de asistencia ventilatoria. Esto se puede explicar porque al implementar una técnica anestésica

Tabla 1. Datos descriptivos de las cirugías.

\begin{tabular}{|llll|}
\hline ASA & $\mathbf{2 - 4}$ & & \\
\hline Diagnóstico $(\mathrm{n})$ & Derrame pleural (15) & Nódulo pulmonar (3) & Empiema (2) \\
Procedimiento $(\mathrm{n})$ & Pleurodesis $(15)$ & Cuña pulmonar (3) & Decorticación pulmonar (2) \\
Duración de la cirugia $(\mathrm{min})$ & $35(15-60)$ & & \\
Duración de la anestesia $(\mathrm{min})$ & $86(50-225)$ & & \\
\hline
\end{tabular}

$(n)=$ número de pacientes. $\min =$ minutos.

Tabla 2. Datos del manejo postoperatorio.

\begin{tabular}{|ll|}
\hline Pacientes en UCI & 10 \\
Promedio dias en UCI & $2(0-15)$ \\
Pacientes en VM (duración en dias) & $2(1-6)$ \\
Pacientes en VMNI (duración en dias) & $3(2-5)$ \\
Pacientes con peridural (duración en dias) & $8(1-3)$ \\
Hospitalización cirugia de tórax (promedio) & $4(1-6)$ \\
Hospitalización total (promedio) & $10(1-30)$ \\
\hline
\end{tabular}

$\mathrm{UCl}=$ unidad de cuidado intensivo. $\mathrm{VM}=$ ventilación mecánica. $V M N I=$ ventilación mecánica no invasiva.
Tabla 3. Complicaciones postoperatorias.

\begin{tabular}{|lll|}
\hline EVA inmediato & $1(1-5)$ & \\
EVA 24 horas & $1(1-4)$ & \\
$\begin{array}{l}\text { Pacientes con necesidad } \\
\text { de opioides }\end{array}$ & 0 & \\
Complicaciones & Falla respiratoria & 3 \\
& Falla renal & 1 \\
& Muerte & 3 \\
\hline
\end{tabular}

EVA $=$ escala visual análoga de dolor. 
nueva los primeros casos realizados con APPD fueron enviados a las unidades de cuidado intensivo para vigilancia respiratoria, situación que fue cambiando y los últimos se trasladaban con tranquilidad a una habitación.

Un hallazgo importante fue el número bajo de complicaciones respiratorias. Similares resultados fueron encontrados por Tacconi y colaboradores (18) en 66 pacientes a quienes les realizaron cirugia de reducción de volumen unilateral con APPD y los compararon con un grupo control con AG. Estos hallazgos van de la mano con el mejor control del dolor postoperatorio y con el hecho de evitar la manipulación de la vía aérea y el uso de ventilación mecánica invasiva.

En cuanto a los resultados obtenidos en relación con la estancia hospitalaria, Pompeo y colaboradores reportaron, en varios estudios para diferentes tipos de procedimientos que incluian resección pulmonar segmentaria (19) metastasectomias (20) y manejo de neumotórax espontáneo (21), que los pacientes manejados con APPD presentaron menor estancia hospitalaria y mayor satisfacción con la técnica anestésica. De la misma manera, Al-Abdullatief y colaboradores (15) demostraron, en un estudio con 79 pacientes, que la APPD disminuye la estancia hospitalaria y la necesidad de $\mathrm{UCl}$ al evitar la AG.

Se requieren estudios con una población más grande que permita comparar aspectos como la estancia en la unidad de cuidado intensivo, el tiempo de ventilación mecánica y en otro tipo de cirugías.

\section{CONCLUSIONES}

La anestesia peridural con el paciente despierto para la realización de cirugias de tórax, es una técnica anestésica segura, que se asocia con baja incidencia de complicaciones respiratorias en el postoperatorio y con disminución del dolor con base en la Escala Visual Análoga evaluada en el posoperatorio inmediato y a las 24 horas postoperatorias. Son precisos más estudios que incluyan mayor número de pacientes y metodologias diferentes para evaluar otras variables.

\section{BIBLIOGRAFÍA}

1. Brodsky J, Lemmens $H$. The history of anesthesia for thoracic surgery. Minerva Anestesiol. 2007; 73: 513-524.

2. Wilson W., Benumof J. Anestesia en cirugia torácica. En: Miller R. Miller Anestesia.. Sexta edición. Madrid: Elsevier; 2005. p. 1847.

3. Galbis J. Anesthesia for thoracic surgery: a challenge for the twenty-first century. Rev Esp Anestesiol Reanim. 2010; 57: 545.

4. Slinger P. Pro: every posthoracotomy patient deserves thoracic epidural analgesia. J Cardiothorac Vasc Anesth. 1999; 13: $350-$ 354.

5. Mineo T. Epidural anesthesia in awake thoracic surgery. Eur $\mathrm{J}$ Cardio-Thorac Surg. 2007; 32: 13-19.

6. Lagunilla J, Garcia J, Fernández A, Álvarez J, Rubio J, Rodríguez J. High thoracic epidural blockade increases myocardial oxygen in coronary surgery patients. Acta Anesthesiol Scand. 2006: $50: 780-786$

7. Loick H, Schmidt C, Van Aken H, Junker R, Erren M, Berendes E, et al. High thoracic epidural anesthesia, but not clonidine, attenuates the perioperative stress response via sympatholysis and reduces the release of troponin $\mathrm{T}$ in patients undergoing $\mathrm{CO}-$ ronary artery bypass grafting. Anesth Analg. 1999; 88: 701-709.

8. Groeben $H$. Epidural anesthesia and pulmonary function. J Anesth. 2006; 20: 290-299.

9. Vanni $G$, Tacconi $F$, Sellitri $F$, Ambrogi V, Mineo T, Pompeo E. Impact of awake videothoracoscopic surgery on postoperative lymphocyte responses. Ann Thorac Surg. 2010; 90: 973-978.

10. Clemente A, Carli F. The physiological effects of thoracic epidural anesthesia and analgesia on the cardiovascular, respiratory and gastrointestinal systems. Minerva Anestesiológica. 2008; 74: $549-563$.

11. Mutz C, Vagts D. Thoracic epidural anesthesia in sepsis - is it harmful or protective. Critical Care. 2009; 13: 182.

12. Yen C, Tsou M, Lin S, Chan K, Chu Y. Thoracic epidural anesthesia for a polymyositis patient undergoing awake mini-thoracotomy and unroofing of a huge pulmonary bulla. Acta Anaesthesiol Taiwan. 2008; 46: 42-45.

13. Groeben H, Schäfer B, Pavlakovic G, Silvanus M, Peters J. Lung function under high thoracic segmental epidural anesthesia with ropivacaine or bupivacaine in patients with severe obstructive pulmonary disease undergoing breast surgery. Anesthesiology. 2002; 96: 536-541.

14. Anderson $M$, Kwong $K$, Furst $A$, Salerno $T$. Thoracic epidural anesthesia for coronary bypass via left anterior thoracotmy in the conscious patient. Eur J Cardio-Thorac Surg. 2001; 20: 415417.

15. Al-Abdullatief M, Wahood A, Al-Shirawi N, Arabi Y, Wahba M, AlJumah $M$, et al. Awake anaesthesia for major thoracic surgical procedures: An observational study. Eur J Cardio-Thorac Surg. 2007; 32: 346-350.

16. Mukaida T, Andou A, Date H, Aoe M, Shimizu N. Thoracoscopic operation for secondary pneumothorax under local and epidural anesthesia in high-risk patients. Ann Thorac Surg. 1998; 65: 924-926.

17. Waurick $\mathrm{R}$, Van Aken $\mathrm{H}$. Update in thoracic epidural anaesthesia. Best Practice and Research Clinical Anaesthesiology. 2005; 19: 201-213.

18. Tacconi F, Pompeo W, Mineo T. Duration of air leak is reduced after awake nonresectional lung volume reduction surgery. Eur $J$ Cardio-Thorac Surg. 2009: 35: 822-828.

19. Pompeo E, Mineo D, Rogliani $P$, Sabato A, Mineo T. Feasibility and results of awake thoracoscopic resection of solitary pulmonary nodules. Ann Thorac Surg. 2004; 78: 1761-1768.

20. Pompeo E, Mineo T. Awake pulmonary metastasectomy. J Thorac Cardiovasc Surg. 2007; 133: 960-966.

21. Pompeo $E$, Tacconi $F$, Mineo D, Mineo T. The role of awake video-assisted thoracoscopic surgery in spontaneous pneumothorax. J Thorac Cardiovasc Surg. 2007; 133: 786-790. 